«En primaria, juegas; acá en secundaria, no». Transición a secundaria en instituciones educativas públicas de Lima

"In primary school, you play. In secondary school, you don't». Transition to secondary education in public schools in Lima.

Flavia Alessandra Demarini Razzeto

Pontificia Universidad Católica del Perú

fdemarini@pucp.pe

Recibido: 16-04-2017

Aprobado: 02-10-2017 


\title{
Resumen
}

El presente estudio cualitativo tiene como finalidad explorar y describir las percepciones de estudiantes de primero de secundaria acerca de su transición a ese nivel educativo, para lo cual se entrevistó a 20 alumnos de 2 colegios públicos de Lima Metropolitana (uno integrado y otro solo de secundaria). La selección de la muestra fue intencional, y a modo de triangulación se realizó un focus group con alumnos de sexto de primaria y entrevistas a profesores de primero de secundaria en ambos colegios. Los resultados muestran que los estudiantes tienen altas aspiraciones educativas y deseo de superación. Sin embargo, la realidad que enfrentan al entrar a secundaria es desfavorable, pues se encuentran con violencia dentro del aula y en los alrededores del colegio, así como con bajas expectativas educativas de los profesores con respecto a su conducta y su rendimiento académico por pertenecer al turno de la tarde. Por otra parte, la ocurrencia simultánea de la transición a la secundaria y a la adolescencia plantea a los alumnos una serie de cambios que deben enfrentar. En esta etapa, los adultos cercanos, como padres y maestros, desempeñan un rol fundamental. No obstante, el estudio encuentra que con frecuencia los padres disminuyen bruscamente el apoyo y la supervisión brindada a sus hijos en comparación con la ofrecida en primaria, a partir de lo cual se pierde la comunicación entre la casa y la escuela. El estudio plantea recomendaciones tanto a nivel del aula y de la escuela, así como de la familia para apoyar a los alumnos en su proceso de transición.

Palabras clave: Transiciones, secundaria, adolescencia, colegios públicos

\begin{abstract}
The objective of this qualitative study is to explore and describe the perception of students that recently made the transition to secondary school. Twenty students from two different public schools in Lima were interviewed (one offered only secondary education and the other one primary and secondary). An intentional sampling method was implemented and in order to triangulate information a focus group with students from last grade of primary school and interviews with teachers of first grade of secondary education were also conducted. The qualitative analysis provided several findings such as the difference between the expectations that the students had when entering secondary school and what they felt in reality. Students have high educational aspirations, although in reality they found bullying in their classes and violence between schools. Furthermore, teachers have lower academic and behavioral expectations of their students given they are attending the afternoon shift. On the other hand, the simultaneous occurrence of the transition to secondary school and adolescence brings several changes for the students. Their significant adults such as parents and teachers play an important role in this stage. Nevertheless, this study shows that they suddenly decrease the support and supervision over the adolescents. Because of that, the communication between home and school also decreases. This study suggests recommendations for the classroom, school and

Keywords: Transitions, secondary school, adolescence, public schools 


\section{«En primaria, juegas; acá en secundaria, no». Transición a secun- daria en instituciones educativas públicas de Lima}

\section{Introducción}

La transición está definida como el paso de una realidad a otra que puede incluir ciertas etapas de crisis e indefinición, pues no se sabe exactamente a dónde se llegará (Sacristán, 1997). Asimismo, se trata de una «experiencia personal y social destacable, no neutra, en la que la identidad se ve alterada y hasta sacudida. En ella no solo nos vemos transformados nosotros mismos, sino también nuestra situación social, los papeles que nos corresponde desarrollar» (Sacristán, 1997, p.17). Implica así un cambio en la persona y en todo lo que rodea a la misma.

Dentro de las transiciones, destacan las educativas, que consisten en pasar de un nivel educativo a otro. En el contexto peruano, resulta interesante e importante estudiar la transición de los estudiantes de primaria a secundaria, puesto que las tasas de deserción y repetición son altas en primero de secundaria: ascienden a un aproximado de 30 mil deserciones anuales a nivel nacional y un promedio de repetición (2010 a 2015) de casi el 6\% de los alumnos matriculados al inicio del año (Ministerio de Educación del Perú, 2016). Adicionalmente, se trata de una transición educativa que se da de manera paralela con la entrada a la adolescencia, ambos cambios significativos en la vida de las personas.

Frente a ello, conocer las percepciones de los propios estudiantes acerca de los retos y dificultades de este período de entrada a la secundaria y a la adolescencia podría ayudarnos a comprender mejor dicha etapa; asimismo, podría ser un insumo para diseñar estrategias para ayudar a los alumnos en este proceso de transición educativa.

\subsection{Contexto personal y social de la transición a secundaria}

A lo largo de la vida, las personas pasan por diversas transiciones (Kagan \& Neuman, 1998, citado en Vogler, Crivello \& Woodhead, 2008), entre las que destacan las educativas, que consisten en el paso de un nivel educativo a otro. En particular, esta investigación se centra en la transición de primaria a secundaria por combinar el hito de pasar a secundaria con una etapa del desarrollo humano como es la entrada a la adolescencia. La adolescencia «se trata de un período marcado por muchos cambios que se reflejan tanto en la apariencia física como en el pensamiento y la vida social» (Giménez-Dasí, 2009, p. 99). Esta combinación puede traer consecuencias tanto positivas como negativas en los estudiantes en relación con su bienestar, su desarrollo psicosocial y su desempeño académico (Seifert \& Schulz, 2007).

Adicionalmente, cabe resaltar la heterogeneidad de los efectos de la adolescencia entre hombres y mujeres. Dado que las mujeres usualmente se 
desarrollan antes que sus pares varones, se puede presumir que es más difícil para ellas el paso a la secundaria (Akos \& Galassi, 2004a). Por ejemplo, el estudio realizado en México por Benjet y Hernández-Guzmán (2002) reportó que alumnos recientes o próximos a hacer la transición a secundaria manifestaron síntomas depresivos asociados, en mayor medida, con aquellos alumnos más entrados en la etapa adolescente. Esto muestra que los cambios internos influenciados por la etapa del desarrollo humano que les toca vivir pueden afectar la percepción sobre la secundaria y sobre el desempeño que tendrán en esta etapa educativa (Elias, 2001).

Un cambio importante que experimentan los adolescentes -entre otroses que van desarrollando su capacidad de autorregulación. Esta es descrita por Zimmerman (1989) como el grado en el que los sujetos son metacognitiva, motivacional y conductualmente activos en su propio proceso de aprendizaje. Aquellos adolescentes autorregulados suelen tener mejores resultados académicos, mayor compromiso con la escuela, mayor aceptación por parte de los padres, adecuada evitación de conductas negativas y patrones más saludables de alimentación (Baummeister \& Vohs, 2007, citado en Bandy, Kristin \& Moore, 2010; Shapiro, 2000, citado en Bandy et al., 2010). En contraste, los adolescentes que no regulan adecuadamente sus emociones y acciones son más propensos a caer en conductas de riesgo (Gestsdóttir \& Lerner, 2007).

De manera paralela, la necesidad de autorregulación viene acompañada por una búsqueda de autonomía que se ve reflejada en la toma de decisiones. Para conseguir mayor autonomía, los adolescentes tendrán que realizar un ajuste de roles y responsabilidades, tanto en el hogar como en la institución educativa (Eccles et al., 1993). El joven busca independencia, mayor libertad y autonomía en la toma de decisiones, y ser tratado como adulto, por lo que la familia debe adaptarse a este nuevo rol (Eccles et al., 1993; Jozefowciz-Simbeni, 2008). Este nuevo papel se modificará también por el cambio corporal que experimentan en esta etapa del desarrollo (Elias, 2001; Benjet \& HernándezGuzmán, 2002; Ellis, Marsh \& Craven, 2009). Para lograr el éxito de los estudiantes en esta etapa de cambios, se requiere del adecuado manejo en casa por parte de los padres y de la participación y del compromiso de estos con la escuela (Woolley \& Bowen, 2007).

\subsection{Principales cambios que enfrentan los estudiantes durante la transición a secundaria}

En relación con las expectativas de los alumnos respecto a su ingreso a la secundaria, los estudios sugieren que, por lo general, existe un enfrentamiento entre estas y la realidad (Chedzoy \& Burden, 2005). Las percepciones sobre la secundaria y los cambios implicados pueden ser positivos en algunos aspectos y negativos en otros, e incluso pueden variar con el paso del tiempo y el momento de la transición en el que están (Ruiz, Castro \& León, 2010). 88 I Al respecto, Wigfield, Byrnes y Eccles (2006) señalan que los alumnos suelen 
percibir dificultad en los cursos, mayor exigencia de los profesores y mayor demanda de tiempo en las tareas. Según estos últimos autores, la percepción usualmente, negativa- de la carga académica podría manifestarse en un menor rendimiento académico, lo que además podría estar relacionado con que se encuentran en una edad en la que el sentido de autoeficacia y la motivación disminuyen (Wigfield et al., 2006). Si bien una vez que ya han pasado a secundaria muchos encuentran un ambiente agradable en el colegio, la carga académica sigue pareciendo bastante elevada (Mackenzie, McMaugh \& O'Sullivan, 2012). Otro problema encontrado es que la secundaria se percibe como un ambiente más estricto y exigente; incluso, existe la expectativa -que se llega a confirmarde que los profesores serán más estrictos que en primaria y la disciplina es percibida como más fuerte (Chedzoy \& Burden, 2005; Akos \& Galassi, 2004b).

Por otra parte, es relevante para los estudiantes que realizan la transición el hacer y mantener amigos, así como encajar en el grupo (Howard \& Johnson, 2004; Akos \& Galassi, 2004b). No contar con el apoyo de los pares podría dar pie a eventos vinculados con el hostigamiento (Akos, 2002), mientras que contar con hermanos mayores que estudian en el centro puede ser positivo, pues se sienten menos amenazados (McKenzie et al., 2012). El área social determinará, en muchos casos, cómo se siente el alumno dentro de la escuela y, por lo tanto, cómo se desempeñará dentro de la misma. Tait (2004, citado en Jozefowciz-Simbeni, 2008) mostró que, durante la adolescencia temprana, hay un aumento significativo del acoso entre compañeros y del rechazo, lo que lleva, en ocasiones, a que los estudiantes se retiren tempranamente del colegio. De este modo, se observa que las relaciones sociales son de tal importancia que podría influir en la decisión de permanecer o abandonar el colegio. En este contexto, comprender estos cambios ayuda a entender cómo se puede mejorar dicha transición (Monarca, Rappoport \& Sandoval, 2013; Calvo \& Manteca, 2016).

Existen algunos estudios longitudinales (Akos, 2002; Akos \& Galassi, 2004b; Chedzoy \& Burden, 2005) que aportan información interesante al encontrar que hay ciertos cambios en las percepciones del grupo conforme pasa el tiempo y según la etapa en la que se encuentran dentro de dicha transición. Sin embargo, en términos del enfoque, son pocos los estudios que ponen énfasis en estudiar la transición a secundaria en el contexto de la transición a la adolescencia y que buscan comprender la influencia de los complejos cambios bio-psicosociales en los procesos educativos.

\subsection{La transición a secundaria en el contexto peruano}

El contexto peruano muestra desigualdad en las oportunidades: existe un colegio público de secundaria por cada cuatro escuelas primarias públicas, una de las mayores tasas de repetición y deserción escolar en primero de secundaria, y una amplia brecha entre la cantidad de estudiantes que terminan la primaria y aquellos que terminan la secundaria (Ministerio de Educación del Perú, 2010). Por ello, el estudio de la transición de primaria a secundaria es un tema 
relevante de análisis. La transición se constituye como un punto de quiebre, en el cual las experiencias de los estudiantes podrían influir, junto con otros factores como el nivel socioeconómico, en su decisión de permanecer o no en la escuela.

En el país, las transiciones dentro de las escuelas han sido principalmente estudiadas desde las Ciencias Sociales (Callirgos, 1995; Ansión, 2005). En particular, la transición a secundaria en el contexto peruano implica que los estudiantes enfrenten una serie de retos:

Desde cambiar de escuela a tener nuevo(s) maestro(s), desde adecuarse a diferentes estilos de enseñanza hasta enfrentar nuevas estructuras organizativas y curriculares, al igual que asumir modificaciones en el ambiente del aula, en el entorno escolar y en las relaciones sociales en este nuevo escenario (Ames \& Rojas, 2011, p. 23).

Los hallazgos, para el caso peruano, en relación con esta transición, no se alejan mucho de lo mencionado en la teoría. Los compañeros representan un gran apoyo para el alumno e incluso llegan a fortalecer su sentido de pertenencia (Cueto, Guerrero, Sugimaru \& Zevallos, 2009). Por esto, el cambio es más difícil si la transición implica también cambiar de institución educativa. Particularmente, en el caso rural, la dificultad va aumentando conforme incrementa la distancia (Ames \& Rojas, 2011). Asimismo, el paso a la secundaria es percibido por los alumnos como algo difícil, pero también es considerado como importante, pues puede determinar el éxito del futuro del estudiante. Estas grandes expectativas, que implican una sensación de cambio para el presente y el futuro de los alumnos, traen consigo una percepción de alta dificultada académica en la secundaria (Ames \& Rojas, 2011).

Por otra parte, en el estudio de Cueto et al. (2009), se ha visto que los estudiantes que perciben un buen sentido de pertenencia al colegio se sienten más cómodos en este y tienen una buena experiencia al pasar a secundaria, debido a que están a gusto con sus profesores, sus compañeros y con la infraestructura de sus escuelas.

Por otro lado, en muchos casos, parece haber hostigamiento en las instituciones educativas, lo que ha llevado incluso a la necesidad de promulgar una ley anti-bullying (Ley 29719) en el Perú. Esta problemática de violencia escolar genera temor, pues los alumnos deben defenderse y quieren contar con un grupo de pares que lo apoye, lo cual es crucial en esta etapa (Akos, 2002; Howard \& Johnson, 2004).

En Perú, los escasos estudios realizados en el tema de transición a secundaria tienen un foco mayor en áreas rurales y no han profundizado en la etapa del desarrollo en la que se encuentran los estudiantes. Resulta relevante estudiar la transición a secundaria tomando en cuenta la etapa del desarrollo humano en la que están estos alumnos y conocer también las características de este proceso en grandes urbes como Lima. 
A la luz de lo anterior, el presente estudio de caso múltiple tiene como objetivo describir las experiencias de estudiantes urbanos de instituciones educativas públicas de Lima que han hecho la transición de primaria a secundaria, y conocer cómo describen su experiencia y cuáles consideran que son los principales retos y dificultades de esta transición. El análisis realizado toma en cuenta que los estudiantes se encuentran además en el proceso de transitar de la niñez a la adolescencia, por lo que busca entender de qué manera la ocurrencia simultánea de ambas transiciones matiza las experiencias de los estudiantes al ingresar al nuevo nivel educativo.

\subsection{Método}

\section{Participantes}

Este estudio de caso múltiple busca conocer las percepciones de los estudiantes que han hecho recientemente la transición a secundaria. Para la muestra intencional, se seleccionó un distrito de Lima Metropolitana: Miraflores, el cual posee más de 7 mil alumnos matriculados de diferentes zonas de la capital en cerca de 15 instituciones educativas públicas (Inei, 2007). Dentro de este distrito, se seleccionaron intencionalmente dos instituciones educativas mixtas y laicas. Una de las instituciones seleccionadas era integrada; y la otra, solo de secundaria. Las dos instituciones contaban con turno mañana y tarde; este último fue elegido para realizar el estudio. Para acceder a estos centros, se llevaron a cabo las gestiones pertinentes con las autoridades educativas del distrito, a quienes se les presentó una carta y un consentimiento informado.

Posteriormente, se realizó la selección intencional de participantes. Para ello, los profesores de primero de secundaria refirieron a cinco alumnos y cinco alumnas bajo la consigna de enviar a sus estudiantes con más alto y más bajo rendimiento académico ${ }^{1}$ con la finalidad de tener en la muestra cierta variabilidad respecto al rendimiento de los alumnos. La edad de la muestra osciló entre los once y catorce años de edad, y seis de los participantes son repetidores en grados de primaria. Ocho de los alumnos provenían de Lima Sur, tres de Lima Centro, y nueve del mismo distrito o distritos contiguos. Por otra parte, tres de los alumnos entrevistados habían finalizado sus estudios de primaria en colegios privados ${ }^{2}$.

1. La consigna dada a los profesores de enviar alumnos con mejor y más bajo rendimiento no fue cumplida a cabalidad, debido a que los profesores tenían la expectativa de que el estudio y la entrevista ayudaran más a aquellos alumnos que no tenían buen rendimiento académico o mala conducta. Por ello, en el balance final, hubo más alumnos con bajo redimiendo académico.

2. En la muestra final, hubo varios alumnos del colegio integrado, primaria y secundaria, que no habían estudiado la primaria en ese colegio, sino que habían cambiado de centro educativo. Parece ser usual el transferirse a otro colegio y, en el caso de este distrito, migrar de un distrito lejano en busca de mejor educación. 
A manera de triangulación de la información y con el objetivo de tener otras perspectivas, se administraron entrevistas semiestructuradas a dos profesores de primero de secundaria de cada institución participante. Finalmente, se realizó un focus group con alumnos de sexto de primaria de la institución educativa integrada. De esta manera, se recabó información acerca de las expectativas que tienen los estudiantes acerca de la transición a secundaria. Este focus group también fue administrado en el colegio a cuatro alumnos con edades entre los diez y doce años $^{3}$. Es importante señalar que los colegios estudiados abarcaron una muestra de casos, por lo que los grados de transferibilidad de los resultados son limitados a contextos muy parecidos al de los colegios estudiados.

\section{Técnicas de recolección de información}

Los instrumentos utilizados fueron entrevistas semiestructuradas y focus group. Estos fueron ajustados tras la realización de una prueba piloto, y aplicados bajo el consentimiento informado de los responsables de las instituciones educativas y previa información a los alumnos y maestros.

Todos los instrumentos recabaron datos generales del entrevistado, percepciones generales sobre la institución educativa y el proceso de transición, percepciones acerca de los principales cambios asociados a la transición y el balance general del proceso de transición a secundaria. Para ello, se empezó con preguntas generales; luego, preguntas más complejas y sensibles; y, finalmente, preguntas de cierre, todas abiertas. Los datos generales del entrevistado permiten entender mejor el contexto y el caso particular de cada estudiante. Con respecto a las percepciones generales sobre la institución educativa y el proceso de transición, se abordaron temas tales como los retos a enfrentar, y el apoyo y el prestigio de la institución educativa. En cuanto a las percepciones sobre los cambios en ciertos aspectos clave, se tomó en cuenta lo encontrado en la revisión de la literatura.

\section{Procedimiento}

El enfoque de investigación utilizado fue el cualitativo, debido a que lo que se buscaba era analizar el discurso de los participantes dentro de su propio contexto y, de este modo, obtener un panorama más amplio acerca de las percepciones de los estudiantes sobre la transición de primaria a secundaria. En lo que respecta al recojo de datos, el levantamiento de información se llevó a cabo durante el segundo semestre del año $2011^{4}$. Cada entrevista individual y

3. La consigna planteada a la profesora fue enviar a dos de sus alumnos con más alto rendimiento y dos con más bajo rendimiento. Fueron enviadas dos niñas y dos niños.

4. El segundo semestre resultaba mejor, pues los estudiantes de primero ya conocían mejor el ambiente de la secundaria, los profesores conocían mejor a sus estudiantes y los alumnos de sexto de primaria estaban cercanos al cambio. 
el focus group duraron aproximadamente 45 minutos. La documentación del proceso fue grabada y transcrita, y tanto las entrevistas como las transcripciones fueron realizadas por el propio investigador.

El procedimiento de codificación se realizó con el software Atlas-ti, y, para ello, se desarrollaron categorías y códigos de análisis. La categorización, basada en dimensiones determinadas, se hizo de manera deductiva guiándose de los principios de la teoría fundamentada. El análisis toma en cuenta diferencias por género, tipo de institución educativa y procedencia de los estudiantes, mientras que los resultados obtenidos fueron discutidos a la luz de la literatura existente antes presentada. Además, se triangula la información obtenida ${ }^{5}$ con miras a incrementar la validez de los hallazgos.

\section{Resultados y discusión}

\section{1 "Quiero ser algo más en la vida». Aspiraciones educativas de los estudiantes en el contexto de la transición a secundaria}

El paso de sexto de primaria a primero de secundaria se encuentra enmarcado en un contexto de motivación por parte de los estudiantes por continuar sus estudios y culminar la secundaria. Esta motivación radica en el deseo de superarse, superar a sus padres y conseguir un futuro mejor estudiando una carrera luego de la secundaria. De este modo, en el discurso de los alumnos, se ve reflejada la idea de la secundaria como un camino para mejorar, que radica en el poder acceder a educación superior en un futuro y cumplir sus metas profesionales.

Porque quiero lograr mis metas; no quiero quedarme como mi mamá. Quiero ser algo más en la vida. Para mí, quedarme en un grado, o sea, no terminar el instituto, la universidad, el colegio y no tener un cartón donde diga «tú eres esto» sería fracasar (alumna, primero de secundaria, colegio solo secundaria, no vive en el distrito).

E: ¿Por qué es importante estudiar?

A: Para ser alguien en la vida.

E: ¿A qué te refieres con alguien en la vida?

A: Para ser abogado o ingeniero (alumno, primero de secundaria, colegio solo secundaria, vive en el distrito).

Tanto en los resultados de este estudio enfocado en alumnos urbanos como el de Ames y Rojas (2011), sobre estudiantes de zonas rurales y urbanas, indican que la importancia de estudiar secundaria radica en el deseo de supe-

5. Se utilizaron entrevistas con alumnos de primero de secundaria, focus group con estudiantes de sexto y entrevistas con docentes. 
ración, lo cual podría estar relacionado con la búsqueda de identidad propia de la adolescencia (Labajos, 1994). Al buscar estudiar algo y «ser alguien en la vida», los alumnos parecen referirse también a encontrar su propia identidad y su rol en la sociedad, con lo que muestran que están pensando en un futuro.

Dado que la mayoría de los alumnos de la muestra provenían de otros distritos y habían estudiado la primaria cerca de sus casas, ahora debían recorrer una mayor distancia. Se pudo observar que el cambio de nivel educativo es aprovechado para realizar un cambio de colegio y de distrito. En este marco, se eligen estos colegios básicamente por la reputación positiva (en términos relativos) que tiene la educación pública en el distrito donde se realizó el estudio. En la mayoría de casos, esto responde a las decisiones familiares; los consejos de padres o hermanos mayores; y, también, al consejo de pares. Solo en algunos casos se debe a una decisión de los propios estudiantes.

E: ¿Querías cambiarte de colegio?

A: Sí.

E: ¿¿Por qué?

A: Mis papás decían que acá era mejor, por la zona y eso (alumna, primero de secundaria, colegio solo secundaria, no vive en el distrito).

Otra estudiante afirma: «No me quedé allá, porque yo quería cambiarme. Quería buscar algo mejor» (alumna, primero de secundaria, colegio integrado, no vive en el distrito).

La distancia llega a ser un tema positivo en el caso de algunos alumnos de la muestra, quienes perciben mayores oportunidades de progreso al movilizarse hacia este distrito y recibir lo que ellos consideran una mejor educación. Resulta destacable la voluntad que tienen para recorrer estas distancias, pues, en sus distritos, sí existe oferta de colegios, a diferencia de los estudiantes de zonas rurales, quienes por falta de oferta se ven obligados a movilizarse grandes distancias para acceder a educación.

Si bien los alumnos entrevistados son conscientes del tiempo que implica movilizarse, muchos aprovechan para ir juntos al paradero o, incluso, hasta sus distritos con compañeros que viven cerca. Esta experiencia de movilizarse juntos les sirve para conversar y verse fuera del colegio, a partir de lo cual afianzan los lazos que pueden servirles como apoyo para la transición sea más fácil. De manera similar, en zonas rurales, el compartir con los compañeros el regreso a casa ayuda a que el sentido de pertenencia a la escuela mejore (Cueto et al., 2009). Es interesante, también, que en áreas rurales (Ames y Rojas, 2011) la transición de primaria a secundaria sea difícil, entre otras cosas, por la distancia entre el lugar en donde se vive y la ubicación del colegio. «Sí, me voy con ellas [mis amigas] juntas, tomamos el mismo carro» (alumna, primero de secundaria, colegio solo secundaria, no vive en el distrito).

Para una minoría este distanciamiento de sus casas les permite además 94 I alejarse de las peleas, o del ambiente agresivo y peligroso que hay en sus barrios 
y en sus colegios anteriores. Tienen una expectativa de que el nuevo ambiente les ofrecerá un entorno menos violento, aunque probablemente esto sea solo relativo.

Sí. Acá es mejor que por allá. [...] mi casa es cerro y se pelean pandillas [...]. Como en mi colegio hay turno mañana y turno tarde, pero en la mañana no me puedo quedar porque he repetido, en la tarde, es un poquito más, más pandilleros, no hago muchas amistades. [...] me gusta la distancia; yo vine para acá por eso. Allá no; no es bueno, mucha pandilla. La distancia es buena; no es la misma gente que allá (alumna, primero de secundaria, colegio solo secundaria, no vive en el distrito).

El estudio realizado encuentra un contraste entre las expectativas de los alumnos y la realidad educativa que enfrentan.

\section{2 «Acá ya no te miran como niña, te miran como más grande». Relaciones con pares y cambios físicos}

Al pasar a primero de secundaria, los alumnos están en una etapa de cambios sociales y físicos debido a la entrada a la adolescencia. Por ello, la mayoría de alumnos, tanto hombres como mujeres, afirmaron que les habría sido más fácil la transición a secundaria si es que sus compañeros de primaria hubiesen seguido estudiando junto con ellos, pues esto habría implicado continuidad y apoyo.

E: ¿Qué lo hubiera hecho mejor [el paso a secundaria]?

A: Que mis amigas vinieran, las de mi otro colegio, pero se quedaron allá (alumna, primero de secundaria, colegio solo secundaria, no vive en el distrito).

A: Que mis compañeros vengan acá (alumno, primero de secundaria, colegio solo secundaria, no vive en el distrito).

Según Ellis et al. (2009) este apoyo podría incluso ayudar a disminuir el estrés de la transición y las conductas de hostigamiento, en la medida que brinda una red de soporte.

Si bien es necesario el apoyo de los pares en esta etapa, hay un cambio importante en las relaciones entre hombres y mujeres que responde al desarrollo físico por el que están pasando. En el caso de ambos colegios, la mayoría de mujeres manifestó notar cambios físicos y personales debido a la edad en la que se encuentran. Tal como Giménez-Dasí (2009) señala, los cambios se dan antes y de manera más notoria en las mujeres, y que esto implica una percepción y experiencia distinta de su transición a secundaria. 
En general, las mujeres notaban que los cambios físicos hacían que la transición se dificultara, puesto que los alumnos mayores podían faltarles el respeto y verlas como mujeres -no como niñas-, por lo cual se sentían más expuestas. Esto se refleja en el comentario de algunas alumnas: «Los grandes [da miedo] cómo te mirarían, porque antes eras chica, pero acá ya no te miran así; te miran como más grande» (alumna, primero de secundaria, colegio solo secundaria, no vive en el distrito). Del mismo modo, otra afirma: «Nos fastidian. No nos meten mano, pero tenemos que defendernos; sé defenderme. Más van y fastidian al colegio de puras mujeres» (alumna, primero de secundaria, colegio solo secundaria, no vive en el distrito).

Por su parte, los pocos alumnos hombres que distinguieron estos cambios físicos y personales debido a la adolescencia señalaron que estos los llevaban a portarse de manera más irrespetuosa, lo que justificaban como un comportamiento adolescente: «He cambiado mi forma de ser: más rebelde. Hay cosas que ya no me gustan tanto, que no aguanto. Me aburro mucho en la clase ahora» (Alumno, primero de secundaria, colegio integrado, no vive en el distrito).

El establecer nuevas relaciones en medio de tantos cambios puede ser difícil para los adolescentes, quienes además extrañan a sus antiguos compañeros $\mathrm{y}$ sienten inseguridad en este nuevo ambiente.

Tal como señalan Benjet y Hernández-Guzmán (2002), dependiendo de cuán desarrollados están los alumnos y alumnas físicamente, estos tendrán una reacción y experiencia distintas de su transición a secundaria. Es importante reconocer y tener en cuenta estas diferencias según género con miras a brindarles a los alumnos un soporte adecuado durante su transición.

\section{3 "Las pandillas que hay acá mucho se pelean». Violencia entre estudiantes dentro y fuera de la institución educativa}

De acuerdo con lo encontrado en este estudio, la diferencia de edad entre los alumnos de hasta cuatro años se debe a las altas tasas de repetición en primero de secundaria, y facilita -junto con otros factores- la existencia de peleas y hostigamiento por parte de alumnos mayores hacia los menores dentro del mismo grado. Esto se observa en los siguientes comentarios de estudiantes: «Porque nos molestan. Es que nosotros somos más pequeños, en primero 'A' hay más repitentes entonces son más grandes. Molestan, nos ponen apodos» (alumno, primero de secundaria, colegio solo secundaria, no vive en el distrito).

[De] primero «E», ese chico mayor como 15 tiene, yo soy «F». Me estaba fastidiando ya con lisura y yo fui, y le metí una cachetada y él quiso levantarme la mano, pues. Yo, por mi reacción, porque soy bien impulsiva y bien nerviosa, y me estaba levantando la mano, yo fui y le metí un cachetadón, y me quiso pegar pues (alumna, primero de secundaria, solo secundaria, no vive en el distrito). 
Estas diferencias de edades parecen generar, en ciertos casos, conflictos ente los alumnos, conflictos que son esperables si se toma en cuenta que toda transición, tanto educativa como del desarrollo humano, implica un grado de ambigüedad de la posición social (Coleman \& Hendry, 2003).

En el caso de los profesores, ellos también observan y reconocen esta situación, así como la problemática que genera el que haya tantos repitentes y diferencia de edades.

E: ¿Se pelean mucho los chicos?

P: Sí.

E: ¿Más en secundaria que en primaria?

P: [...] más que primaria, pero no tanto. Yo ahorita tengo a $1^{\circ} \mathrm{D}$ y la gran mayoría ahí, no hay muchos, hay 22 nada más, pero la mayoría de ahí son repitentes. Ya tienen 13, 14; entonces, yo digo mucha diferencia de edad (Profesora de primero de secundaria, colegio integrado).

Algunos conflictos implican poca confianza entre los compañeros y fastidiarse de manera verbal: «Porque hay cosas como que te ocultan, te fastidian o hablan a tus espaldas mal» (Alumna, primero de secundaria, colegio solo secundaria, no vive en el distrito). Otros conflictos, en cambio, implican agresiones físicas: «[...] se pelean a golpes» (Alumna, primero de secundaria, colegio integrado, no vive en el distrito).

E: ¿Lo que menos te gusta del colegio?

A: Los malcriados, los que pelean (Alumno, primero de secundaria, colegio integrado, vive en el distrito).

Los alumnos manifiestan que existe hostigamiento dentro del colegio y muchas peleas entre los colegios de la zona, lo que genera que se sienta cierta inseguridad dentro y fuera del colegio: «A veces me gustaría ver (las peleas), pero me da miedo que me caiga una piedra o algo» (alumna, primero de secundaria, colegio solo secundaria, no vive en el distrito).

Según la información recogida en este estudio, parece haber dos niveles de agresión: uno en que los insultos predominan y suelen darse dentro de la institución educativa, y otro nivel en el que resaltan las agresiones físicas entre alumnos de distintos colegios. No obstante, los maestros no parecen estar informados sobre las peleas al interior de la institución educativa o minimizan el problema. En cambio, las peleas contra otros colegios sí son vistas como algo grave por los profesores, por lo cual afirman que, cuando suceden, los alumnos son sancionados con suspensiones y, en ocasiones, se cita a los padres, aunque no siempre asisten.

Muchos alumnos entrevistados afirmaron que, al ver una pelea, preferían pasar desapercibidos y miraban por curiosidad; sin embargo, una minoría de estos observadores señaló que avisan a un profesor cuando presencian o se 
enteran de un acto de violencia. Esto podría explicar la falta de información de los docentes acerca de estas peleas.

La información acerca de la violencia en secundaria fue corroborada por los alumnos de sexto de primaria, quienes afirmaron tener temor de pasar a secundaria, especialmente, debido a los alumnos mayores y los posibles maltratos que podrían recibir por parte de estos últimos, más aún en el turno de la tarde.

$\mathrm{Al}$ respecto, uno de los alumnos afirma: «Y cuando estás ahí los de quinto cuidan a los de primero cuando no está el profesor, entonces te amenazan en momentos» (focus group con alumnos de sexto de primaria, colegio integrado). De este modo, se observa que existe temor hacia la violencia en el colegio, principalmente, hacia los alumnos mayores, a partir de lo cual la secundaria es percibida como un ambiente amenazante. Dicha información es encontrada en otros estudios realizados (Akos, 2002; Akos \& Galassi, 2004a), en los que se señala que la percepción de la secundaria como un lugar en el que podría darse el maltrato por parte de alumnos mayores es una constante. Este miedo a ser maltratados podría estar afectando las percepciones de los estudiantes y generándoles estrés al encontrarse con un clima escolar en el que no se sienten seguros (Ellis et al., 2009; Treviño et al., 2010).

Dentro de esta problemática, el pertenecer al turno de la tarde, como es el caso de los participantes de este estudio, resulta ser un agravante más. Al turno de la tarde suelen acudir aquellos alumnos que tuvieron un bajo rendimiento académico o mostraron dificultades en conducta durante la primaria. Por esto, en ambos colegios, está mal visto por los maestros y por los alumnos menores el pasar a este turno, mostrándose temor hacia posibles conductas inadecuadas y teniendo una expectativa de fracaso en el ámbito académico.

E: ¿Qué saben del turno de la tarde?

Alumno 1: Es bien peligroso. Si yo paso al turno de la tarde, me cambio. Alumna 2: Yo me cambio también si paso a la tarde.

[...]

E: ¿Por qué no quieren la tarde?

Alumna 3: Es que yo una vez vine para el recreo de la tarde y se tiran todo. Son muy malcriados, hablan lisuras y todo (focus group con alumnos de sexto de primaria, colegio integrado).

Los propios alumnos de primero de secundaria mencionan que el estar en turno tarde no es necesariamente una decisión de ellos. Más bien, verbalizan su malestar por la desigualdad de oportunidades que enfrentan en comparación con sus pares del turno de la mañana. Alegan que este turno desorganiza sus horarios; incluso, algunos llegan a usarlo como justificación del que no hagan las tareas, puesto que en las mañanas deben ayudar en sus casas o duermen. Sobre este turno, una estudiante afirma: «[...] en mi colegio, hay turno mañana y turno tarde, pero en la mañana no me puedo quedar, porque he repetido. En la 
tarde, es un poquito más, más pandilleros; no hago muchas amistades» (alumna, primero de secundaria, colegio solo secundaria, no vive en el distrito). A su vez, otro alumno señala:

En turno mañana creo que hay más orden. Veo que nadie se pelea. Salen del colegio y se van a su casa; en cambio, acá salen del colegio y se ponen en las esquinas a conversar. Por eso, se pelean contra otros colegios (alumno, primero de secundaria, colegio integrado, no vive en el distrito).

Si bien en el discurso de los estudiantes -analizado anteriormente- hay una motivación por mejorar, las posibilidades que se les brindan al entrar a este turno de tarde no son las mejores, debido a que se cuenta con una expectativa de fracaso por parte del entorno, y son alumnos que en general tienen un bajo rendimiento académico o mala conducta. En el turno tarde, deben enfrentar un ambiente de peleas entre colegios e incluso dentro de la propia institución educativa, o cuentan con poco apoyo social, realidades que podrían resultar poco motivadoras.

Cabe señalar que una de las características de los adolescentes es la rebeldía, el no aceptar la autoridad y el no querer depender de nadie (Labajos, 1994). Frente a ello, es indispensable que el colegio busque mecanismos para apoyar a los alumnos en esta transición tomando en cuenta las características propias de esta etapa.

\section{4 "Más difícil es acá». Percepción de los estudiantes sobre los cambios académicos al ingresar a secundaria}

A partir de esta investigación, se puede observar que las responsabilidades de la secundaria se contrastan con las libertades y momentos divertidos y tranquilos que había en la primaria. Estos cambios son notorios en el área académica, la cual cobra más peso que en la primaria, debido a la necesidad de una adecuada preparación para «ser alguien en la vida». Esto último va cobrando mayor importancia por encontrarse en una etapa del desarrollo en que se va forjando la identidad y se piensa en el futuro. Al respecto, una estudiante señala: «Es distinto, como que en secundaria te enseñan diferente; en primaria, uno juega más» (alumna, primero de secundaria, colegio solo secundaria, no vive en el distrito). En esa misma línea, otra alumna afirma: «En la primaria, juegas mucho y hablas cosas, de juguetes. Acá, en secundaria, no» (alumna, primero de secundaria, colegio solo secundaria, no vive en el distrito).

En este estudio, se encontró que la expectativa de la mayoría de alumnos antes de pasar a secundaria era que la carga académica sería más difícil y confirmaron, una vez iniciada la secundaria, que efectivamente lo era. 
Tengo que estudiar un poquito más. Antes yo estudiaba, daba una leidita y ya. Ahora tengo que buscar [...] y tengo que investigar. [...] No es como la primaria, que tú pensabas y te ponían "Ah ya»; tienes que poner una idea que caiga con la tarea. Acá te lo revisan (alumna, primero de secundaria, colegio integrado, no vive en el distrito).

Para otros alumnos, incluso, las exigencias superaron sus expectativas de dificultad: «[...] fue más difícil de lo que pensé [pasar a secundaria]. Está difícil [...] algunas veces dejan poca tarea, pero igual es difícil. Yo no pensé que iba a hacer tantas responsabilidades» (alumno, primero de secundaria, colegio solo secundaria, no vive en el distrito).

Solo unos pocos no anticipaban la secundaria como un nivel más difícil y, según lo presentado en su discurso, no les parecía más complicado en cuanto a lo académico: «En el colegio que yo estaba, era particular y eran tranquilos; acá todos son movidos. [...] Más fácil es acá. No tengo que estudiar tanto; en el otro, sí, porque era particular» (alumno, primero de secundaria, colegio integrado, vive en el distrito). En relación con ello, se debe considerar que, en el caso de alumnos provenientes de colegios particulares, su percepción sobre la transición, al menos en el punto de dificultad académica, parece ser distinta que la de sus pares que han estudiado siempre en colegio público. Es posible que la imagen positiva que algunos colegios particulares puedan tener en el país, en comparación a la educación pública, esté afectando esta percepción.

Para los alumnos de primero de secundaria, la transición a dicho nivel educativo se ve dificultada por el poco apoyo académico y emocional que les brindan sus profesores. De acuerdo con lo señalado por los estudiantes, una gran diferencia entre primaria y secundaria es que en primaria tenían un solo profesor que los conocía bien, los apoyaba emocional y académicamente, y con quien tenían una relación más cálida y cercana. Asimismo, percibían el aspecto académico como algo fácil y más recreacional. Varios participantes afirmaron que se les hubiese hecho más fácil la transición a secundaria si sus profesores de primaria hubiesen continuado con ellos por la confianza que tenían con estos, así como la atención y el apoyo para ayudarlos a resolver problemas personales y académicos. En secundaria, en cambio, los alumnos señalaban que tener tantos profesores les hacía sentir que estos no se conectaban con ellos, pues ni siquiera conocían sus nombres. Adicionalmente, cada profesor tenía estilos diferentes de enseñanza y para los estudiantes era difícil adaptarse a ello.

Sí, te dejan un montón de tareas, todos los profesores piensan que su curso es el único, y te dejan harta tarea y mucho pues [...]. [En primaria] Tuve dos profesores más o menos. Uno con varios cursos. Luego, otro para inglés, pero no dejaban tanta tarea. Ellos sabían cuánto dejar; ya sabían, pues. Acá son muchos profesores; todos te dejan tarea. No saben si hay de otro curso también; te dejan todo (alumno, primero de secundaria, colegio solo secundaria, no vive en el distrito). 
De manera similar, otra estudiante indica: «Primaria ha sido más, más - cómo se llama- jugar y te explicaban; secundaria es diferente. Te enseñan más profesores, viene un profesor y viene otro, te confunden y dejan un montón de tarea» (alumna, primero de secundaria, colegio integrado, no vive en el distrito).

Como consecuencia del mayor número de profesores, los alumnos reportaban que otros retos o dificultades asociados a la transición consistían en que ahora tenían que enfrentar nuevos cursos y que, en general, tenían más tareas que en primaria. Según lo señalado por los estudiantes, ellos tienen gran dificultad para comprender muchos de los temas vistos en clase y sienten que sus profesores no siempre les explican hasta que entienden. Algunos de ellos manifestaron que esta situación podía llevarlos a reprobar un curso. No obstante, algunos pocos alumnos mencionaron a ciertos maestros que sí se preocupaban por ellos, y los motivaban y les daban segundas oportunidades, lo que ayudaba y facilitaba el proceso de cambio.

Esto se refleja en los comentarios de los estudiantes: "Ahora es más difícil» (alumno, primero de secundaria, colegio solo secundaria, no vive en el distrito). Otro alumno señala: «Sí, hay que estudiar más. No explican tanto y antes en primaria era solo teoría; ahora, acá también hay prácticas. [...] Ahora tú tienes que responder y sin que te expliquen mucho» (alumno, primero de secundaria, colegio solo secundaria, no vive en el distrito). En un tercer testimonio, se afirma: "Que a veces hay cosas muy difíciles que no entiendo y le preguntas a los profesores y te dicen "ya un ratito", "después". Pero no te responden nunca» (alumno, primero de secundaria, colegio solo secundaria, no vive en el distrito).

Los adolescentes deberían haber alcanzado, o estar próximos a alcanzar, una estructura de pensamiento formal, lo que debería permitirles tener un razonamiento hipotético-deductivo y resolver problemas con mayor facilidad (Giménez-Dasí, 2009). No obstante, les parece muy difícil, pues probablemente o bien aún no alcanzan este estadio del pensamiento o no se organizan de manera adecuada, y la forma autoritaria de los profesores no está ayudándolos. A pesar de que supuestamente hay un aumento de madurez, suele suceder que los profesores de grados mayores imparten mayor disciplina y control que los de primaria, y proveen a los estudiantes de menos oportunidades para ejercer la toma de decisiones y aprender a autorregularse (Feldlaufer et al., 1988, citado en Eccles y Roeser, 2010).

Según lo encontrado en este estudio, los profesores entrevistados también percibían las dificultades académicas de los estudiantes y comentaban que sus alumnos no dominan todos los contenidos académicos que deberían.

Académico son muchas cosas; por ejemplo, cosas que ven en primaria ya no se acuerdan. Por ejemplo, los acentos, palabras agudas ya no se acuerdan. Muchos se olvidan. En cuanto a la madurez, también, están mucho a la broma, al chiste, al chisme, que me dijeron esto, lo otro. 
[...] Algunos ni su nombre lo escriben bien; se olvidan de la mayúscula (profesora de primero de secundaria, colegio solo secundaria).

Los alumnos de sexto de primaria también manifestaron preocupaciones sobre la mayor dificultad académica del nivel secundaria y afirmaron no sentirse preparados para este paso.

E: ¿Hay algo que les de miedo de pasar a secundaria?

A: Sí, las clases van a ser más difíciles.

[...]

E: ¿Sienten que están preparados para pasar a secundaria?

A1: Más o menos.

A2: No (focus group con alumnos de sexto de primaria, colegio integrado).

Las dificultades académicas identificadas anteriormente podrían contribuir a explicar las altas tasas de repetición en primero de secundaria. Tal como Chedzoy y Burden (2005) afirman, los retos académicos debido a la dificultad de la secundaria son uno de los mayores desafíos que enfrentan al pasar de nivel educativo. Sin embargo, en opinión de los profesores entrevistados, el alto número de repitentes en primero de secundaria no solo estaría relacionado con el aspecto académico, sino también con lo que ellos consideran «inmadurez» de los adolescentes para poder afrontar la secundaria: «Hay algunos chicos bien incapaces [...]. Cada vez se ha ido incrementando más [la cantidad de repitentes]; de eso, me doy cuenta. En cambio, antes no sé por qué los chicos eran más maduros» (profesora de primero de secundaria, colegio integrado).

A la luz de lo señalado por los diferentes actores entrevistados, parecería que los estudiantes no se encuentran preparados académicamente para responder a los cambios y los retos asociados con su transición a secundaria. Si bien esto podría depender hasta cierto punto de las capacidades del propio alumno, sería pertinente analizar si existe una continuidad entre el currículo o si salen de primaria sin cumplir los objetivos planteados. Adicionalmente, se podría sugerir un acompañamiento personalizado por parte de la escuela y los padres para conseguir el éxito académico o un proceso más paulatino de cambio de nivel educativo.

Otro tema importante es el concepto negativo que tienen los profesores de los alumnos, especialmente, hacia los del turno tarde y la influencia de esto en la conducta de los últimos. En una etapa de formación de identidad, como es la adolescencia, la idea de otros sobre el adolescente podría influenciar en él y su comportamiento (Rosenthal \& Jacobson, 1968). El desempeño es mejor cuando los estudiantes están social, emocional e intelectualmente apoyados por los profesores (Eccles \& Roeser, 2010). 


\title{
2.5 «Sus papás no se enteran, pero los míos sí». El rol de la familia en el proceso de transición
}

Las dificultades y los retos que enfrentan los alumnos se enmarcan en el contexto de la entrada a la adolescencia. El perfil de niños se va dejando atrás, y se asume que son más responsables y autónomos, lo que hace que sus padres y maestros ya no los traten como en primaria, sino que les otorgan más responsabilidades y libertad esperando que asuman su autonomía correctamente.

\begin{abstract}
E: ¿Antes también tenías esas responsabilidades?
A: No, recién. Ahí me engreían en primaria; ahora pasé a secundaria y ya tengo que ayudar más, además de que tengo que estudiar más, porque hay mucha más tarea y eso. [...] Cumplir con las tareas; de ahí, cuidar a mi hermana cuando estoy en mi casa; las tareas del hogar también. Las tareas del colegio y de la casa (alumno, primero de secundaria, colegio solo secundaria, no vive en el distrito).
\end{abstract}

Si bien se espera que los adolescentes asuman una serie de responsabilidades cuando pasan a secundaria, se debe destacar que el apoyo de los padres, igual que el de maestros, cambia. Dicho cambio podría estar dándose, porque los padres tienen menos control en las acciones de sus hijos, debido a que estos se movilizan solos y se van muchas horas lejos al colegio. Los padres se ven en la necesidad de adaptarse a los nuevos roles de sus hijos; sin embargo, el cortar el apoyo tan drásticamente, en vez de acompañar y de enseñar a enfrentar las nuevas responsabilidades, parece dificultar la transición para los alumnos, quienes afirman que la falta de apoyo de sus padres y el tener menos supervisión por parte de estos los ha afectado académica y conductualmente. En general, la supervisión por parte de los padres parece disminuir ahora que han pasado a secundaria: «Antes estaba en turno mañana y ya pues me ayudaban a veces; ahora no. Hago mis cosas en la mañana y mi mamá ya no tiene tiempo, y son cosas más difíciles también» (alumno, primero de secundaria, colegio integrado, no vive en el distrito). De este modo, se puede observar que, en relación con el aspecto académico, los alumnos perciben menor apoyo, a pesar de estar entrando a una etapa en la que -como se vio- la carga académica es más exigente. Sin embargo, también se espera que tengan mayor autonomía, la que no parecen manejar adecuadamente o no han adquirido.

De acuerdo con la percepción de los propios alumnos, la disminución de la supervisión se relaciona con el hecho de que han crecido y deben asumir mayores responsabilidades, por lo que sus padres dejan de prestarles la atención de antes. Al no tener supervisión en su casa, pueden tener más libertad para tomar algunas decisiones, como faltar a clases ${ }^{6}$. Los resultados de esta muestra

6. Muchos alumnos de la muestra no vivían con ambos padres e, incluso, algunos vivían con otros familiares, por lo que se dificulta la supervisión parental en algunos casos. 
indican que a la mayoría de adolescentes no les atemorizan las sanciones del colegio, sino las que podría haber en sus hogares, pero saben que es poco probable que las haya, pues sus padres no se enteran de sus faltas. Los alumnos que no faltan a clases afirmaron que su mayor temor era que sus padres se enteraran y que estaban seguros de que, si faltaban, lo sabrían, puesto que ellos les revisan siempre el cuaderno de control y se mantenían en contacto con los profesores. Esto permite, además, observar que sí hay padres que supervisan a sus hijos, y muestra que los alumnos supervisados son aquellos que usualmente tienen mejor desempeño académico y conducta según lo que ellos mismos y los profesores revelan.

[A] Los que no les hacen nada sí faltan, pues. No están sus papás, no saben si faltan; si yo falto, me pegan. Pero ellos no están nadie en la casa, y ya no pasa nada ni saben si vinieron o no, porque no les ven el cuaderno de control. A veces, me dicen para tirarme la pera, pero yo digo no. Es que sus papás no se enteran, pero los míos sí y yo no quiero (alumna, primero de secundaria, colegio integrado, no vive en el distrito).

En general, al ser percibidos como mayores por parte de la familia, los padres asumen un rol más distante. La falta de supervisión ocasiona que los alumnos no hagan tareas en la casa, ya sea porque no tienen a quién pedirle ayuda o bien porque saben que nadie los sancionará en casa si es que no hacen la tarea. Esta falta de vigilancia se encuentra estrechamente ligada a la baja autorregulación de los estudiantes, quienes sin una supervisión externa parecen fallar en el cumplimiento de sus responsabilidades (Zimmerman, 2000).

En lo que respecta a las percepciones de los estudiantes, esta varía: se reconocen con cierta capacidad de autonomía, dejan de autopercibirse como niños $\mathrm{y}$ reconocen las nuevas responsabilidades que deben asumir.

Como estoy en secundaria y soy un poquito más grande, como que tengo que fijarme más en mis cosas, más en mis tareas. No es como en primaria, que yo podía estarle diciendo a mi mamá que me ayude; acá tengo que hacer sola mis cosas (alumna, primero de secundaria, colegio solo secundaria, no vive en el distrito).

A pesar de sentirse como mayores y plantearse ciertas metas, como el terminar la secundaria para tener un futuro mejor, muchos de estos alumnos no llegan a tener una adecuada autorregulación para alcanzar sus metas aún.

E: ¿Por qué faltas al colegio?

A: A veces, me aburro.

E: ¿A dónde vas cuando faltas?

A: A internet, a jugar. 
E: ¿Vas solo o con otros compañeros?

A: Con mis compañeros; ellos me dicen para ir (alumno, primero de secundaria, colegio integrado, vive en el distrito).

La falta de autorregulación en estos alumnos podría deberse a que estaban habituados a una regulación externa. Podría ser cuestionable, entonces, el apoyo que se da en primaria para preparar a los alumnos para secundaria y para que aprendan a asumir responsabilidades, tanto por parte de los profesores de primaria como por parte de los padres. También, se evidencia que hay una influencia del grupo de pares.

Es importante señalar que algunos alumnos sí consiguen regularse, ya sea por factores internos - como el querer superarse y tener un buen rendimiento-, o por factores externos -como el evitar un castigo por parte de la escuela o los padres-, lo que parece ser en ciertos casos más fuerte que la presión del grupo de pares: «Me molesta porque distraen. El otro día me han dicho para ir al Internet, tirarnos la pera. [...] pero no quiero porque de ahí se enteran (alumno, primero de secundaria, colegio solo secundaria, no vive en el distrito).

Los estudiantes entrevistados para el presente estudio reportan que es frecuente que los adolescentes falten a clases para ir a fiestas, cabinas de Internet o simplemente a pasear. Se podría decir que los adolescentes no parecen manejar adecuadamente la libertad y responsabilidad que se les otorga, debido a que no cuentan con suficiente autorregulación, a lo que se suma la supervisión de los adultos que los rodean, que es baja.

La falta de supervisión en casa y la disminución de apoyo de los docentes en comparación con primaria genera una ruptura en la comunicación entre la casa y el colegio. Esta falta de comunicación que podría ayudar a brindar cierto soporte a los estudiantes se ve obstaculizada por la distancia que hay entre la casa y la escuela, la cual no permite muchas veces que los padres puedan asistir a las reuniones programadas. También, se hace más complicada esta comunicación y supervisión si se compara con la primaria, período en que la mayoría de estos alumnos tenían pocos o incluso solo un profesor, quienes los conocían bien y podían hacer un seguimiento más cercano.

Al haber un cambio en los roles, sin haber una adecuada comunicación entre la escuela y la familia del estudiante, el apoyo académico y emocional hacia este parece disminuir en ambos ambientes. Para los profesores queda en evidencia que los alumnos que no reciben apoyo familiar suelen tener mayores problemas de conducta y académicos. Además, los maestros entrevistados indicaron que la falta de comunicación entre la institución educativa y la casa responde a que los padres toman cierta distancia de los alumnos y del colegio.

Varios son de familias disfuncionales [...]. El papá trabaja, la mamá trabaja y los dejan solos todo el día; entonces, ya no hacen la tarea y así se la pasan. Tenemos un chico que ha estado faltando, o tenía tardanza o no venía. Después de vacaciones, los hemos citado y hemos averiguado 
[...]. «cuando llego a mi casa mi mamá me agarra a correazos y me dice regresa», entonces, él regresa y a dónde se va: se va a los parques, por acá, a Internet, esperando a que sean las seis de la tarde (profesora de primero de secundaria, colegio integrado).

Frente a ello, toman medidas extremas, como no dejar entrar a clases a los alumnos hasta que los padres se presenten a las reuniones pactadas: «Se les cita [a los papás]. Se tiene que insistir. Ya cuando ocurre un hecho de que ya prácticamente no se permite, ya se le hace una suspensión por dos días o un día, dependiendo del caso» (profesor de primero de secundaria, colegio integrado).

En las entrevistas, incluso, se afirmó que algunos padres llegan a otorgar absoluta responsabilidad a la escuela hasta el punto de mencionar que aceptan que se agreda a sus hijos físicamente si eso es necesario para que obedezcan.

Lo que piden los papás es que nos dan permiso de que les peguemos; eso nos dicen. Antes [nos lo pedían] más; este año ha bajado. Ellos se quejan, cuestionan que uno no les grita, que no somos fuertes con ellos. Después, todas las denuncias que hay (profesora de primero de secundaria, colegio integrado).

El dejar de contar con el apoyo de la familia y maestros, en algunos casos, podría llevar a problemas académicos y conductuales, debido a que desmotiva a los estudiantes, puesto que muchos parecen encontrarse motivados extrínsecamente para evitar el castigo. En ese sentido, los alumnos no parecen estar preparados para asumir muchos de los retos que enfrentan al realizar estas transiciones; continúan rigiéndose por los beneficios o castigos que pueden recibir si realizan o no una tarea, por lo que necesitan constantemente de un apoyo externo que los ayude a justificar sus conductas. De acuerdo con la teoría del desarrollo moral de Kohlberg (1981), se esperaría que los adolescentes se encuentren en un estado convencional, en que la orientación moral se rige por las relaciones sociales y por el orden social; sin embargo, estos alumnos parecen estar en un estado preconvencional aún, en el que basan sus decisiones $y$ conductas según el beneficio que obtengan o el castigo que eviten.

\section{Balance final}

Los resultados muestran los principales retos y dificultades de esta transición educativa que se combina con la entrada a la adolescencia. El estudio encuentra un claro deseo de superación de los adolescentes y una alta valoración de la educación, no solo a nivel de discurso, sino también demostrada mediante acciones concretas. Sin embargo, a pesar de estas grandes expectativas, lo que ellos encuentran en muchos casos es una realidad poco favorable. En primer lugar, se observa que la alta tasa de repetición en primero de secundaria genera que en los salones haya alumnos con edades muy diferentes y es común que los 
mayores abusen de los menores. En segundo lugar, los alumnos del turno tarde enfrentan un ambiente educativo complejo, en el que hay bajas expectativas tanto por parte de los profesores como de los mismos alumnos, debido a que en ese turno suelen concentrarse los estudiantes con más dificultades académicas y conductuales.

Por otro lado, en este nuevo nivel, los alumnos deben afrontar cambios sociales: ocupar un nuevo rol, y asumir nuevas responsabilidades y libertades, tanto en el colegio como en otros contextos. Dentro de estos cambios, los adultos cercanos, como padres y maestros, desempeñan juegan un rol fundamental. Los resultados han mostrado que estos adultos disminuyen el apoyo, en relación con el que daban en primaria, de manera casi inmediata. Los padres dejan de supervisar a sus hijos, y los maestros reducen el apoyo académico y emocional comparado con el que se les brindaban en primaria. Asimismo, el nivel de coordinación entre la casa y la escuela disminuye; en la medida que el alumno percibe esta falta de comunicación, se siente más libre para tomar decisiones. No obstante, los adolescentes no siempre saben manejar la libertad que se les otorga de manera adecuada, y toman decisiones como faltar a clases o entrar en peleas, pues sienten que no habrá sanciones. Frente a ello, hace falta un seguimiento mayor por parte de los adultos que acompañan paulatinamente en este cambio a los estudiantes, quienes, al estar en el inicio de la adolescencia, se encuentran pasando por muchos cambios que no logran manejar adecuadamente sin apoyo. Incluso, académicamente, no parecen estar preparados, según lo que perciben los maestros y alumnos, lo que causa que esta etapa sea más difícil y, por lo tanto, requiere de mayor apoyo.

Esta investigación contribuye a la literatura sobre transición aportando una mirada desde la psicología a la ocurrencia simultánea de dos transiciones importantes en la vida de las personas - a la adolescencia y la educación secundaria- detallando los respectivos retos a nivel personal, social y académico que esto supone, y que han sido especificados anteriormente. Pensando en una futura agenda de investigación, sería interesante estudiar este fenómeno en otros contextos. Por ejemplo, podría contarse con una población dividida en colegios mixtos y segregados, de modo tal que pueda profundizarse en los temas relacionados con la maduración de los alumnos según el género de los mismos. Finalmente, se podría realizar entrevistas a los padres, de modo que ellos puedan dar su punto de vista, puesto que se ha visto que cumplen un rol importante en esta transición. En términos del diseño, sería enriquecedor poder estudiar las percepciones de los estudiantes sobre la transición usando un diseño longitudinal, de manera tal que se puedan analizar los cambios y continuidades en el discurso de los estudiantes.

Del presente estudio, también, se desprenden recomendaciones. En primer lugar, es importante trabajar el clima del aula. El apoyo de los pares es clave en el proceso de transición, y, sin embargo, entre los alumnos de secundaria se reportan con frecuencia episodios de hostigamiento y violencia, frente a lo cual es fundamental que las instituciones educativas tomen cartas en el asunto. 
Se debe trabajar el tema de acoso escolar con todos los actores implicados: alumnos, profesores y padres de familia, de manera que pueda prevenirse e intervenirse. En segundo lugar, se debería desarrollar, dentro de las instituciones, sistemas para identificar alumnos en riesgo, como podrían ser aquellos que tienen poco apoyo en casa, y brindarles el apoyo y el seguimiento que requieren. Finalmente, para que esta transición sea más fácil, es indispensable fortalecer la relación entre las familias y la institución educativa. Con el apoyo y la supervisión necesarias por parte de los adultos, tanto padres como profesores, el ausentismo escolar y los problemas académicos podrían disminuir. Para ello, se podría brindar herramientas a los estudiantes para conseguir una mejor autorregulación, y realizar una transición más pausada y no tan drástica como parece ser que sucede.

Por último, cabe señalar como limitaciones de este estudio el haber contado con participantes, en su mayoría, provenientes de otros distritos al de su escuela. Esto se da particularmente en el distrito estudiado, debido a que es percibido como un distrito con mejor calidad académica. Sería interesante contrastar las percepciones de alumnos de otros distritos con los del presente estudio. Además, es probable que, de acuerdo con lo señalado por los propios estudiantes, haya diferencias entre las percepciones de aquellos que asisten al turno de la mañana, pues a este suelen ir los alumnos con mejor rendimiento académico e incluso conducta.

\section{Agradecimientos}

Agradezco a los alumnos y docentes de ambas instituciones por el tiempo dedicado y disposición para las entrevistas, así como a los directores por la confianza y permiso que me otorgaron para realizar el estudio. Asimismo, agradezco a Gabriela Guerrero por su continuo apoyo, paciencia y dedicación puestos en sus correcciones y comentarios.

\section{Nota biográfica}

\section{Flavia Demarini Razzeto}

Es profesora de la Facultad de Psicología de la Pontificia Universidad Católica del Perú y de la Maestría en Cognición, Aprendizaje y Desarrollo de la misma casa de estudios. Además se desempeña como psicóloga en un colegio de Lima. Es Magíster en Psicología Educativa por la Universidad de Barcelona. Se especializa en la investigación sobre primera infancia y desarrollo humano. Ha publicado Competencias genéricas en los Estudios Generales Letras con lAugusta Valle (Lima: PUCP, 2017) y Atención y educación de la primera infancia en el Perú: avances y retos pendientes con Gabriela Guerrero (Lima: GRADE, 2016). 


\section{Referencias}

Akos, P. (2002). Student perceptions of the transition from Elementary to Middle School. Professional School Counseling, 5(5), 339- 345.

Akos, P. \& Galassi, J. (2004a). Gender and race as variables in psychosocial adjustment to Middle and High School. The Journal of Educational Research, 98(2), 102-108.

(2004b). Middle and high school transitions as viewed by students, parents, and teachers. Professional School Counseling, 7(4), 212- 222.

Ames, P. \& Rojas, V. (2011). Cambios y oportunidades: La transición de la escuela primaria a la secundaria en el Perú. Niños del Milenio. Documento de trabajo 63. Lima: Grade.

Ansión, J. (2005). Discriminación y pluralismo cultural en la escuela: Lima Cusco, Perú. En P. Marambio (Ed.), Discriminación y pluralismo cultural en la escuela. Casos de Brasil, Chile, Colombia, México y Perú. Santiago: Unesco / Orealc.

Bandy, T., Kristin, B. S. \& Moore, A. (2010). Assessing self regulation: A guide for out-of-school time program practitioners. Washington D.C.: Child Trends. Recuperado de: http://www.childtrends.org/Files//Child_ Trends-2010_10_05_RB_AssesSelfReg.pdf

Benjet, C. \& Hernández-Guzmán, L. (2002). A short-term longitudinal study of pubertal change, gender and psychological well-being of Mexican early adolescents. Journal of Youth and Adolescence, 31(6), 429-442. doi: 10.1023/A:1020259019866

Callirgos, J. C. (1995). La discriminación en la socialización escolar. Lima: Pontificia Católica del Perú.

Calvo, A. \& Manteca, F. (2016). Barreras y ayudas percibidas por los estudiantes en la transición entre la educación primaria y secundaria. Reice. Revista Iberoamericana sobre Calidad, Eficacia y Cambio en Educación, 14(1), 49-64.

Chedzoy, S. M. \& Burden, R. L. (2005). Making the move: Assessing student attitudes to primary-secondary school transfer. Research in Education, 74, 22-35.

Coleman, J. C. \& Hendry, L. B. (2003) Psicología de la adolescencia (4a ed). Madrid: Ediciones Morata.

Cueto, S., Guerrero, G., Sugimaru, C. \& Zevallos, A. (2009). Sense of belonging and transition to high schools in Peru. International Journal of Educational Development, 30(3), 277-287.

Eccles, J.,y Roeser, W. (2010). An ecological view of schools and development. In J. Meece \& J. Eccles (Eds.), Research on schools, schooling and human development. New York: Routledge. 
Eccles, J. S., Midgley, C., Wigfield, A., Buchanan, C. M., Reuman, D., Flanagan, C. \& Mac Iver, D. (1993). Development during adolescence: The impact of stage-environment fit on young adolescents' experiences in schools and in families. American Psychologist, 48(2), 90-101. doi: 10.1037/0003066X.48.2.90

Elias, M. J. (2001). Easing transitions with social-emotional learning. Principal Leadership, 1(7), 20-26.

Ellis, L., Marsh, H. \& Craven, R. (2009). Addressing the challenges faced by early adolescents: A mixed-method evaluation of the benefits of peer support. American Journal of Community Psycholy, 44(1), 54-75. doi 10.1007/s10464-009-9251-y

Gestsdóttir, S. \& Lerner, R. (2007). Intentional self-regulation and positive youth development in early adolescence: Findings from the 4-h study of positive youth development. Developmental, 43(2), 508-521. doi: 10.1037/0012-1649.43.2.508

Giménez-Dasí, M. (2009). El desarrollo del pensamiento. En B. Delgado Egido (Coord.), Psicología del Desarrollo: Desde la infancia hasta la vejez (pp. 97114). Vol. 2 (2 $2^{\mathrm{a}}$ ed.). Madrid: McGraw-Hill.

Howard, S. \& Johnson, B. (2004). Transition from primary to secondary school: Possibilities and paradoxes. In AARE 2004, Conference Papers. HOW04184.

Instituto Nacional de Estadística e Informática (Inei) (2007). Mapa de pobreza provincial y distrital 2007. Censo Nacional 2007-INEI-Progama de las Naciones Unidas para el Desarrollo (PNUD) Perú 2007. Lima: Inei.

Jozefowciz-Simbeni, D. M. H. (2008). An ecological and developmental perspective on dropout risk factors in early adolescence: Role of school social workers in dropout prevention efforts. Children \& School, 30(1), 49-62.

Kohlberg, L. (1981). Essays on moral development. Vol. I: The philosophy of moral development: Moral stages and the idea of justice. San Francisco: Harper y Row.

Labajos, J. A. (1994). Identidad del adolescente. En A. Aguirre Baztán (Ed), Psicología de la adolescencia. Barcelona: Marcombo, S.A.

Mackenzie, E., McMaugh, A. \& O’Sullivan, K. (2012). Perceptions of primary to secondary school transitions: Challenge or threat? Issues in Educational Research, 22(3), 298-314.

Ministerio de Educación del Perú (2010). Censo Escolar del Ministerio de Educación-Unidad de Estadística Educativa. Lima: Minedu. Recuperado de: http://escale.minedu.gob.pe/indicadores2010.

(2016). Censo Escolar del Ministerio de

Educación-Unidad de Estadística Educativa. Lima: Minedu. Recuperado de: http://escale.minedu.gob.pe/indicadores2016 
Monarca, H., Rappoport, S. \& Sandoval, M. (2013). La configuración de los procesos de inclusión y exclusión educativa. Una lectura desde la transición entre educación primaria y educación secundaria. Revista de Investigación en Educación, 11(3), 192-206.

Rosenthal, R. \& Jacobson, L. (1968). Pygmalion in the classroom: Teacher expectation and pupils' intellectual development. Nueva York: Holt, Rinehart y Winston.

Ruiz, L., Castro, M. \& León, A. (2010). Transición a la secundaria: Los temores y preocupaciones que experimentan los estudiantes de primaria. Revista Iberoamericana de Educación, 52(3), 1-13.

Sacristán, G. (1997). La transición a la educación secundaria. Madrid: Morata.

Seifert, T. \& Schulz, H. (2007). The effects of pubertal timing and school transition on preadolescents's well-being. Canadian Journal of School Psychology, 22(2), 219-234.

Treviño, E., Valdés, H., Castro, M., Costilla, R., Pardo, C. \& Donoso Rivas, F. (2010). Factores asociados al logro cognitivo de los estudiantes de América Latina y el Caribe. Santiago de Chile: Salesianos Impresores S.A. Serce/ Unesco.

Vogler, P., Crivello, G. \& Woodhead, M. (2008). La investigación sobre las transiciones en la primera infancia: Análisis de nociones, teorías y prácticas. Cuadernos sobre Desarrollo Infantil Temprano $N^{\circ} 48$. La Haya: Fundación Bernard van Leer.

Wigfield, A., Byrnes, J. \& Eccles, J. (2006). Development during early and middle adolescence. In P. A. Alexander y P. H. Winne (Eds.), Handbook of educational psychology. Mahwah, NJ: Lawrence Erlbaum Associates.

Woolley, M. \& Bowen, G. (2007). In the context of risk: Supportive adults and the school engagement of middle school students. Family Relations, 56(1), 92-104. doi: 10.1111/j.1741-3729.2007.00442.x

Zimmerman, B. J. (1989). A social cognitive view of self-regulated academic learning. Journal of Educational Psychology, 81(3), 329-339.

(2000). Attaining self-regulation: A social cognitive perspective. In M. Boekaerts, P. R. Pintrich \& M. Zeidner (Eds.), Handbook of self-regulation. San Diego, CA: Academic Press. 
